\title{
An Improved PSO Algorithm for Direction Finding with Tetrahedral-based USBL System
}

\author{
Jian Huang ${ }^{*}$ and Shenggang Yan \\ School of Marine Science and Technology, Northwestern Polytechnical University, Xi'an, China \\ *Corresponding author
}

\begin{abstract}
Aiming at the problem that the standard particle swarm optimization algorithm has a slow convergence speed and easy to fall into the local optimum, the method of region-division is introduced to dynamically adjust the inertia weight and learning factors of the particle to achieve the balance between optimization ability and convergence speed, and adaptive mutation operation are used to avoid the population falling into the local optimum. The proposed algorithm is applied to the target direction finding by a tetrahedral-based ultra-short baseline positioning system. Simulation experiments show that the proposed algorithm can effectively improve the accuracy of direction finding and achieve the purpose of accelerating the convergence of PSO algorithm.
\end{abstract}

Keywords-particle swarm optimization; ultra-short baseline; region-division; adaptive

\section{INTRODUCTION}

The ultra-short baseline (USBL) positioning system is a commonly used underwater acoustic positioning system, which is attributed to its low price, easy to install and operate and having a certain accuracy. The core problem of USBL system design is to create a reliable system that can accurately determine the position of underwater objects relative to the USBL system under real ocean conditions[1-3]. The USBL system calculates the distance and bearing angle of the target by measuring the Time-of-Arrival (TOA) and TimeDifference-of-Arrival (TDOA) of the acoustic signal propagation.

The operating conditions of the USBL system may be very unsatisfactory. The existence of environmental and system noise, multipath effect, refraction of sound rays and other factors can significantly reduce the positioning accuracy of USBL system, and may cause abnormal operation of the system. To overcome these difficulties is the subject of recent researches to improve the accuracy and stability of USBL system. The latest researches in this field mainly focus on the development of modern signal processing methods and the design of receiving elements for USBL system[4-7]. One of the most promising methods is to place the receiving elements more efficiently to resolve the ambiguity of the object position and avoid the situation where the target positioning accuracy is poor due to the positional relationship between the object and the receiving elements of the USBL system.

A planar array can locate the target in the entire space or in a half space bounded by the plane of the array, which can met the system requirements in many cases. Chen et al.[8] studied the positioning principle of planar arrays and analyzed the system error in detail. Min et al.[9] and Xiang et al.[10] proposed an 8-element planar array, and effectively solved the phase difference ambiguity problem by increasing the number of elements. ARKHIPOV M.[11, 12] introduces the design of a 3-D USBL system receiving array, where the improving accuracy and stability is achieved by positioning the target by using a complete set of the elemental (three-element) USBL arrays.

The TDOA based Direction-of-Arrival (DOA) estimator performs data processing in the time domain. The azimuth angle and elevation angle of the target are calculated directly from the estimated TDOA and do not need the time-consuming 2-D search over the array manifold as the beamformer does, therefore, TDOA technology is more efficient at calculating load[2]. Currently, the Least Squares (LS) algorithm is the most important method of DOA estimation based on TDOA[13], however, the LS algorithm can achieve the ideal performance only when the array is geometrically symmetric. The performance of the Maximum Likelihood (ML) based method can reach the theoretical bound, but requires more stringent conditions. Evolutionary algorithms are swarm-based intelligent optimization methods, some of which have been studied for DOA estimation and positioning. Zaman et al.[14,15] proposed a Genetic Algorithm (GA) hybridized with pattern search for the joint amplitude and DOA estimation using a special array of L-type shape. Lui et al.[16] used Particle Swarm Optimization (PSO) to locate the signal source based on TDOA measurement. In [17], the GA and PSO algorithm are used to estimate the target direction using TDOA measurement on a 3-D acoustic sensor array.

In order to further improve the positioning accuracy of the USBL system, we propose an improved PSO algorithm for tetrahedral-based USBL system positioning. In order to improve the convergence speed of the standard PSO algorithm and solve the premature convergence problem, we proposes the following improvements: the population is divided into different regions according to the distance between each particle to the global optimum. The particles adaptively adjust their inertia weights and learning factors by different strategies to achieve the balance between optimization ability and convergence speed, and the adaptive mutation operation is introduced to avoid the population falling into the local optimum. Simulation experiments show that the proposed algorithm can effectively improve the positioning accuracy and stability of USBL system. 


\section{USBL POSITIONING}

Acoustic signal propagates underwater in the form of spherical waves, and their curvature will affect the TDOA measurement. However, considering that the length of the array baseline is much smaller than the distance between the target and the USBL system, the arrival wave can usually be approximated as a plane wave (PW). Morgado et al.[18] studied the positioning problem in the reference coordinate system based on PW and spherical interpolation (SI) method, with the conclusion that PW technology is more effective and less sensitive to sensor noise than SI method. This conclusion holds as long as the ratio between the baseline length and the slant range of the target is greater than $4 \%$.

Therefore, we assume that the operating environment of the USBL system is in compliance with far-field conditions. At the same time, we also assume that the transducer on the target is time synchronized and that the processing delay of the transceiver is known or negligible. The average sound speed of the acoustic signal in the water is known and the received signal is unaffected by multipath effect.

To simplify the description, we only consider the problem of how to determine the position of the underwater target relative to the USBL system. The coordinate system shown in Figure 1 is established with the center of the USBL system as the origin. Suppose there are $M$ receivers, and their coordinates are expressed as $\mathbf{p}_{m}=\left[x_{m}, y_{m}, z_{m}\right]^{T}$, where $m \in\{1,2, \ldots, M\}$. The acoustic signal arrives at the USBL system array with azimuth angle $\varphi$ and elevation angle $\theta$. Define $\mathbf{s} \in \mathbf{R}^{3}$ as the normalized direction vector point to the direction of the target.

$$
\mathbf{s}=[\cos (\varphi) \cos (\theta), \sin (\varphi) \cos (\theta), \sin (\theta)]^{T}
$$

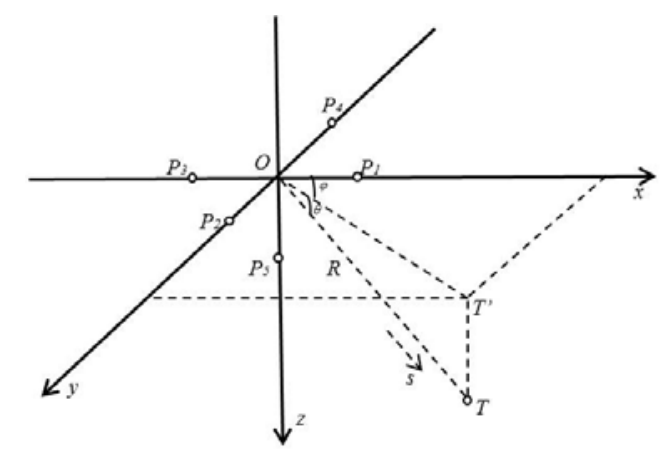

FIGURE I. POSITIONING PRINCIPLE OF USBL SYSTEM

According to the PW approximation, the true value of TDOA between a pair of receivers is given by:

$$
\tau_{i j}=t_{i}-t_{j}=-\frac{1}{c} \mathbf{s}^{T}\left(\mathbf{p}_{i}-\mathbf{p}_{j}\right), \quad i, j \in M, i \neq j
$$

where $t_{m}$ is the TOA at the receiver $m$, and $c$ is the sound speed. Collect all possible TDOA values between receiver pairs into a single vector:

$$
\Delta=\left[\tau_{1,2}, \tau_{1,3}, \cdots, \tau_{(M-1), M}\right]^{T}
$$

then we have,

$$
\Delta^{T}=-\frac{\mathbf{s}^{T} \mathbf{D}^{T}}{c}
$$

where

$$
\mathbf{D}=\left[\begin{array}{c}
\left(\mathbf{p}_{i}-\mathbf{p}_{j}\right)^{T} \\
\left(\mathbf{p}_{i}-\mathbf{p}_{j}\right)^{T} \\
\vdots \\
\left(\mathbf{p}_{i}-\mathbf{p}_{j}\right)^{T}
\end{array}\right] \in \mathbf{R}^{k \times 3}
$$

where $k$ is the total number of pairs of $M$ receivers, given by $k=M \cdot(M-1) / 2$.

Let the measured value of TDOA is $\hat{\tau}_{i, j}$, and the vector consists of all possible TDOA measurements between receiver pairs is expressed as:

$$
\hat{\Delta}=\left[\hat{\tau}_{1,2}, \hat{\tau}_{1,3}, \cdots, \hat{\tau}_{(M-1), M}\right]^{T}
$$

Thus, we define the following cost function by the difference between the measured values of TDOA and their true values:

$$
f(\theta, \varphi)=\|\hat{\Delta}-\Delta\|_{2}
$$

where $\|\cdot\|_{2}$ represents the 2-norm of the vector. The purpose of the DOA estimator based on PSO algorithm is to minimize $f(\theta, \varphi)$ according to $\theta$ and $\varphi$ and find its global optimal solution.

\section{IMPROVED PSO}

\section{A. standard PSO}

The mathematical description of the standard PSO is that there are $N$ particles in a $D$-dimensional search space (solution space), and each particle is regarded as a search individual in the search space. Each particle has its own position and velocity vector, and the vector size depends on the parameters involved in the optimization problem. In each iteration of PSO, the particle updates its position by tracking its historical optimal solution and the global optimal solution, and updates its velocity and position vector by (8) and (9), respectively.

$$
\boldsymbol{v}_{I d}^{k+1}=\omega \boldsymbol{v}_{I d}^{k}+\eta_{1} r_{\text {rand } 1}^{k}\left(\boldsymbol{p}_{\text {bestId }}^{k}-\boldsymbol{x}_{\text {Id }}^{k}\right)+\eta_{2} r_{\text {rand } 2}^{k}\left(\boldsymbol{g}_{\text {best }}^{k}-\boldsymbol{x}_{\text {Id }}^{k}\right)
$$




$$
\boldsymbol{x}_{I d}^{k+1}=\boldsymbol{x}_{I d}^{k}+\boldsymbol{v}_{I d}^{k+1}
$$

where, $\boldsymbol{v}$ represents the velocity vector, $\boldsymbol{x}$ represents the position vector, and the subscript $I d$ is the particle identifier; $\omega$ is the inertia weight; and $\eta_{1}$ and $\eta_{2}$ are the learning factors in the range of $[0,4]$, usually take $\eta_{1}=\eta_{2}=2 ; r_{\text {rand } 1}$ and $r_{\text {rand } 2}$ are uniformly distributed random floating point numbers within [0, 1] that give the particle the ability to move within the search space. $\boldsymbol{p}_{\text {bestld }}$ is the optimal position vector that the particle has obtained so far, and $\boldsymbol{g}_{\text {best }}$ is the global optimal position vector of the entire population.

\section{B. Improved PSO}

In many improvement of PSO algorithms[19-22], accelerating the convergence speed and avoiding falling into the local optimal solution are the main research directions. Based on the standard PSO algorithm, we introduce regiondivision based on the Euclidean distance between particles, and divides the population into two parts according to its Euclidean distance from the global optimal particle. The particles closer to the global optimal particle focus on enhancing its local search ability, while the particles farther away from the global optimal particle focus on enhancing its global search ability. On this basis, we give the improvement strategies as follows.

\section{1) Region-division based on Euclidean distance}

In the $i$-th iteration, define $L_{i j}$ as the Euclidean distance between $j$-th particle and the global optimal particle, which can be calculated as:

$$
L_{i j}=\sqrt{\sum_{k=1}^{D}\left(g_{b e s t k}-p_{j k}\right)^{2}}
$$

where $D$ represents the dimension of the search space, $g_{\text {best }}$ and $p_{j k}$ represents the component of the global optimal particle and the $j$-th particle in the $k$-th dimension, respectively.

All particles are sorted according to the Euclidean distance from the global optimal particles. The entire search space is divided by a limit $L_{\lim }$ (the selection of $L_{\mathrm{lim}}$ have to ensure that the number of particles satisfying $L_{i j} \leq L_{\text {lim }}$ reaches a certain proportion, for example, $40 \%$ of the population) into two parts: the inner region which contains "good" particles; and the outer region which contains "poor" particles.

\section{2) Adaptive adjustment of inertia weight and learning} factors

In (8), $v_{I d}^{k}$ represents the flight speed of the particle at previous moment, $\boldsymbol{p}_{\text {bestld }}^{k}$ and $\boldsymbol{g}_{\text {best }}^{k}$ represent the "selfexperience" and "social experience" of the particle, respectively. It can be seen that the flight trajectory of the particle is affected by both the inertia weight and the learning factors. The inertia weight $\omega$ controls the effect of the historical moment on the current state, which can balance the global search ability and local search ability of the particle. Larger inertia weight help to enhance global exploitability (larger search range), while smaller inertia weight tend to perform local searches at the current location. $\eta_{1}$ and $\eta_{2}$ then determine the influences by the individual optimum and the global optimum.

Based on the region division of the search space, we expect that the "good" particles have better local search ability, so as to achieve better local search within the inner region; at the same time, the "poor" particles located in the outer region can have better global search ability, in order to find potential better regions, so as to avoid the population falling into local optimal solution. On this basis, we design a linear strategy to adaptively adjust the inertia weight and learning factors of each particle. Let $i$ and $j$ denote the $i$-th iteration of the population and the $j$-th particle, respectively. $t_{i}$ represents the $i$-th iteration, $t_{\text {max }}$ represents the total number of iterations. $L_{\max }$ and $L_{\min }$ represent the maximum and minimum values of the Euclidean distance between any particle and the global optimum, respectively. The inertia weight and learning factors are calculated as follows:

$$
\omega_{i j}=\left\{\begin{array}{l}
\omega_{\min }+\frac{t_{\max }-t_{i}}{t_{\max }} \cdot \frac{\left(L_{i j}-L_{\mathrm{lim}}\right) \cdot\left(\omega_{\max }-\omega_{\min }\right)}{L_{\max }-L_{\mathrm{lim}}}, L_{i j}>L_{\mathrm{lim}} \\
\omega_{\min }+\frac{t_{\max }-t_{i}}{t_{\max }} \cdot \frac{\left(L_{i j}-L_{\mathrm{min}}\right) \cdot\left(\omega_{\max }-\omega_{\min }\right)}{L_{\mathrm{lim}}-L_{\min }}, L_{i j} \leq L_{\mathrm{lim}}
\end{array}\right.
$$

$$
\eta_{1 i j}=\left\{\begin{array}{l}
\eta_{\min }+\frac{\left(L_{i j}-L_{\mathrm{lim}}\right) \cdot\left(\eta_{\mathrm{max}}-\eta_{\mathrm{min}}\right)}{L_{\mathrm{max}}-L_{\mathrm{lim}}}, L_{i j}>L_{\mathrm{lim}} \\
\eta_{\min }+\frac{\left(L_{i j}-L_{\mathrm{min}}\right) \cdot\left(\eta_{\mathrm{max}}-\eta_{\mathrm{min}}\right)}{L_{\mathrm{lim}}-L_{\mathrm{min}}}, L_{i j} \leq L_{\mathrm{lim}}
\end{array}\right.
$$

$$
\eta_{2 i j}=\left\{\begin{array}{l}
\eta_{\max }-\frac{\left(L_{i j}-L_{\mathrm{lim}}\right) \cdot\left(\eta_{\mathrm{max}}-\eta_{\mathrm{min}}\right)}{L_{\mathrm{max}}-L_{\mathrm{lim}}}, L_{i j}>L_{\mathrm{lim}} \\
\eta_{\max }-\frac{\left(L_{i j}-L_{\mathrm{min}}\right) \cdot\left(\eta_{\max }-\eta_{\min }\right)}{L_{\mathrm{lim}}-L_{\min }}, L_{i j} \leq L_{\mathrm{lim}}
\end{array}\right.
$$

In (11), $\omega_{i j}$ is the inertia weight of the $j$-th particle in the $i$ th iteration, $\omega_{\max }$ and $\omega_{\min }$ are the maximum and minimum values of the inertia weight, respectively, where $\omega_{\max }=0.9$ and $\omega_{\min }=0.4$. For the particles in the outer region, the farther it is away from the global optimum, the better global exploitation ability it has; for the particles in the inner region, the closer it is to the global optimum, the better local search ability it has. The component $\frac{t_{\max }-t_{i}}{t_{\max }}$ ensures that all particles have better regional search capabilities in the later stages of the iterations. 
In (12) and (13), $\eta_{1 i j}$ and $\eta_{2 i j}$ represent the learning factors of the $j$-th particle in the $i$-th iteration, respectively. $\eta_{\max }$ and $\eta_{\min }$ are the maximum and minimum values of the learning factors, respectively, where $\eta_{\max }=3$ and $\eta_{\min }=1$. For particles in the outer region, the farther it is away from the global optimum, the more it is affected by "self-experience" (ie, the larger value of $\eta_{1}$ ), and the less it is affected by "social experience" (ie, the smaller value of $\eta_{2}$ ). For particles in the inner region, the closer it is to the global optimum, the less it is affected by "self-experience" and the more it is affected by "social experience".

\section{3) Adaptive mutation}

Based on the above improvements, the convergence speed and accuracy of the PSO algorithm have been greatly improved, but there is still possibility of falling into local optimum and unable to jump out. therefore, we propose the adaptive mutation operation.

First, we determine whether the particles are concentrated in a very small region, and the criterion is to satisfy one of the following two conditions [19]:

a) $\boldsymbol{g}_{\text {best }}$ has no improvement in successive $b$ iterations, the ideal value of $b$ is:

$$
5 \% \leq \frac{b}{t_{\max }} \leq 10 \%
$$

b) Define the Euclidean distance between the particle $j$ to the global optimal particle is $L_{g j}$, and the distance function between all the particles and the global optimum as:

$$
s(L)=\sum_{j=1, j \neq g}^{N} \frac{1}{\pi L_{g j}}
$$

which satisfies:

$$
s(L)<t_{i} \cdot N \cdot \sqrt{D} \cdot e^{a}
$$

where $a$ is a constant between [0,1].

The premise of the mutation operation is that the particle satisfies the condition of the concentrated minimum region, and the mutation probability is decided by its Euclidean distance to the global optimum, calculated as follows:

$$
p=d \cdot\left(\frac{L_{\max }-L_{i j}}{L_{\max }-L_{\min }}\right)
$$

where, $d$ is a constant between $[0,1]$ which is used to adjust the range of the mutation probability. The smaller the Euclidean distance between the particles and the global optimum, the greater the probability of mutation. For particle $p_{i j}$, a random number $r$ between $[0,1]$ is generated, and if it is satisfies $r \leq p$, the particle performs the mutation operation. First, a particle $p_{i k}$ in the out region is randomly selected, and then perform the mutation operation according to the following strategy.

$$
\boldsymbol{x}_{i j}^{n e w}=\boldsymbol{x}_{i j}+r \cdot\left(\boldsymbol{x}_{i j}-\boldsymbol{x}_{i k}\right)
$$

where, $\boldsymbol{x}_{i j}$ and $\boldsymbol{x}_{i k}$ are the position vectors of the particles $p_{i j}$ and $p_{i k}$, respectively. $\boldsymbol{x}_{i j}^{\text {new }}$ is the position vector of particle $p_{i j}$ after the mutation operation.

\section{Algorithm process}

The USBL positioning algorithm based on improved PSO can be summarized as follows:

Step 1: Enter the known parameters, define the required variables and randomly generate a population of $N$ particles, randomly initialize the position and velocity of each particle;

Step 2: Calculate the fitness of each particle, and store the historical optimum of each particle and the global optimum, respectively;

Step 3: Calculate the distance between each particle and the global optimum according to (10), and divide the population into inner and outer regions;

Step 4: Determine whether the population is concentrated in a very small region, if so, perform the mutation operation and go to Step 6, otherwise go to Step 5;

Step 5: For each particle, calculate its inertia weight and learning factors according to (11) (13);

Step 6: Update the velocity and position of each particle according to (8) and (9);

Step 7: Determine whether the algorithm satisfies the stopping criterion. If yes, proceed to the next step, otherwise go to Step 2;

Step 8: Output the global optimum, that is, the azimuth angle and elevation angle of the target.

\section{SimUlations}

The performance of the proposed method was tested by simulation experiments. The experimental environment is as follows: CPU is Intel Core i7-4710MQ; RAM is DDR3L-8GB; operating system version is: Windows 8.1; Matlab version is R2014a.

The parameters of the simulation have the following values: the sound speed in the water is set to a constant $c=1500 \mathrm{~m} / \mathrm{s}$; the arrangement of the array elements is shown in Figure 1, and the distance between each array element and the origin is set to $0.15 \mathrm{~m}$. In each run, the measured value of TDOA is calculated by the following equation. 


$$
\hat{\tau}_{i, j}=\frac{R_{j}-R_{i}}{c}+\varepsilon_{i, j}
$$

where $\varepsilon_{i, j}$ is the additive white Gaussian noise with mean value of 0 and a standard deviation of $\sigma_{\tau}$.

When using the improved PSO (iPSO) based algorithm proposed in this paper, the search space of the particle is limited to $0^{\circ} \leq \varphi \leq 180^{\circ}, 0^{\circ} \leq \theta \leq 90^{\circ}$, and the position vector of the particle is randomly initialized in a uniform distribution manner. The range of particle velocity is set to [-2, 2]. The number of particles is set to 100 and the maximum number of iterations is set to 100 . Other parameters are set as $a=0.5, b=$ $7, d=0.7$.

Monte Carlo simulations were performed to evaluate the proposed USBL positioning algorithm given different DOA values under PW approximation. Assuming that the signal reception conditions allow us to measure the time delay without error, the measurement accuracy is limited only by the signal sampling frequency, so it is assumed that $\sigma_{\tau}=0.5 / f_{s}$, where $f_{s}=200 \mathrm{kHz}$ is the sampled frequency. We assume the slant range to the target is $R=1000 \mathrm{~m}$. During the simulation, the azimuth angle changes from $0^{\circ}$ to $170^{\circ}$, the elevation angle changes from $0^{\circ}$ to $80^{\circ}$. The azimuth angle and elevation angle are all changing by step of $3^{\circ}$, then we have a total of 1624 sampling points. For each point, 20 random experiments were performed and the azimuth angle and elevation angle are calculated using the LS algorithm and the iPSO based algorithm, respectively, and the mean and standard deviation of positioning error were calculated for the estimation results. The experimental results are shown in Figure 2 and Figure 3. It can be seen from Figure 2 and Figure 3 that the iPSO based positioning algorithm can achieve the purpose of improving the positioning accuracy.

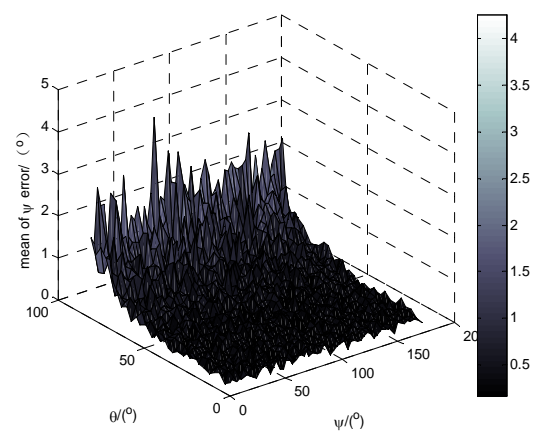

Figure II. (a) mean of $\varphi$ error

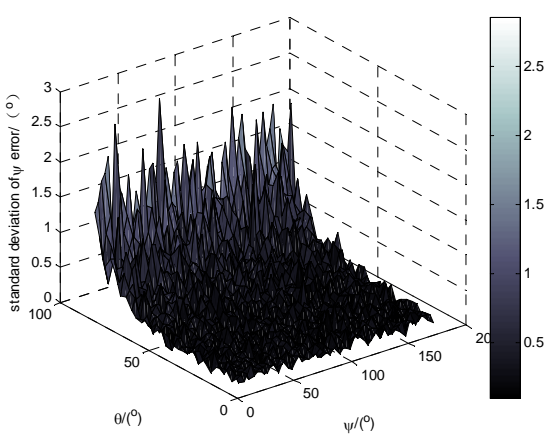

Figure II. (b) standard deviation of $\varphi$ error

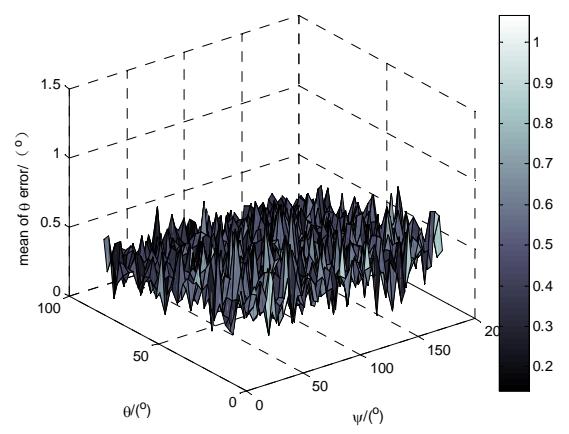

Figure II. (c) mean of $\theta$ error

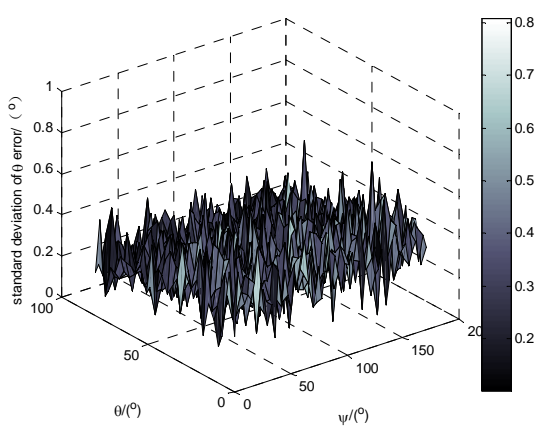

Figure II. (d) standard deviation of $\theta$ error

FIGURE II. POSITIONING RESULTS OF LS ALGORITHM

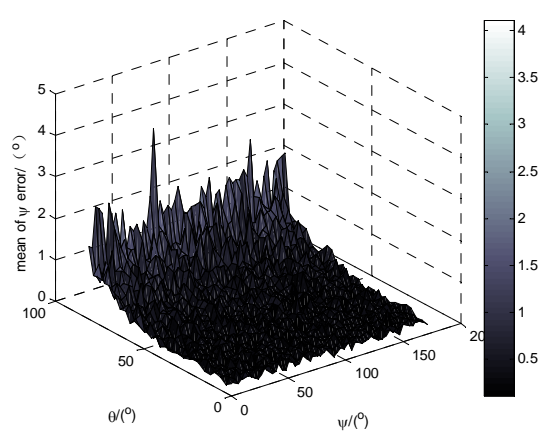

Figure III. (a) mean of $\varphi$ error 


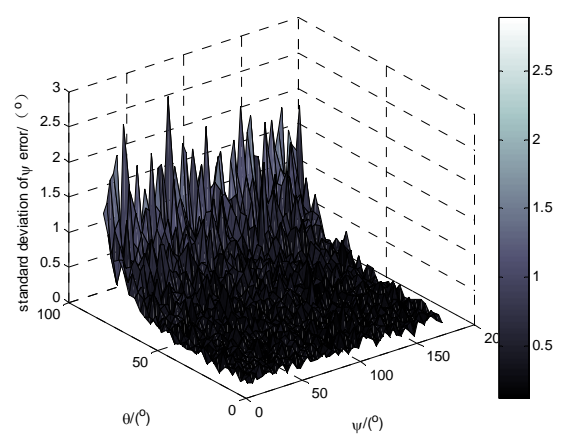

Figure III. (b) standard deviation of $\varphi$ error

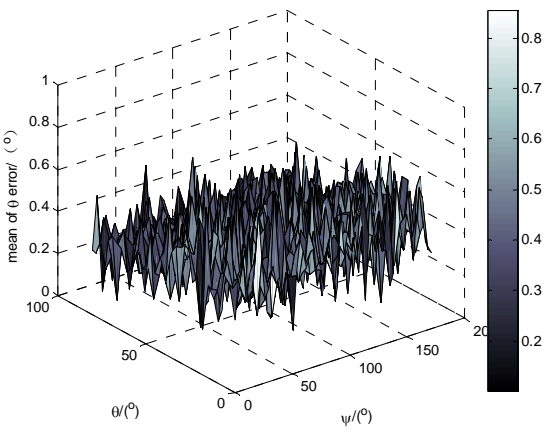

Figure III. (c) mean of $\theta$ error

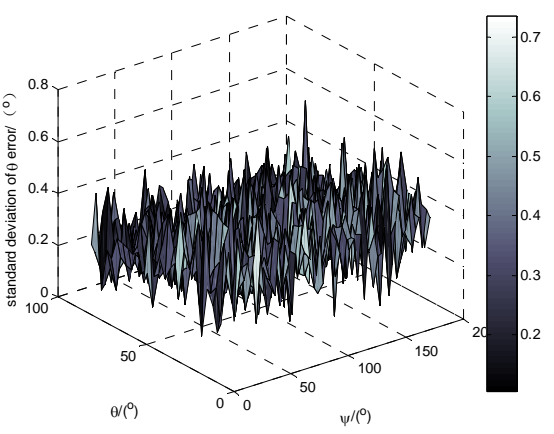

Figure III. (d) standard deviation of $\theta$ error

FIGURE III. POSITIONING RESULTS OF IPSO ALGORITHM

Repeat the above simulation to test the effect of noise intensity on the two methods by changing the value of $\sigma_{\tau}$. The average values of the DOA estimation error of each algorithm under different noise conditions are calculated. The test results are shown in Figure 4. It can be seen that the DOA estimation error of iPSO based algorithm has lower value at any noise conditions, which means that iPSO based method has stronger stability against noise influence.

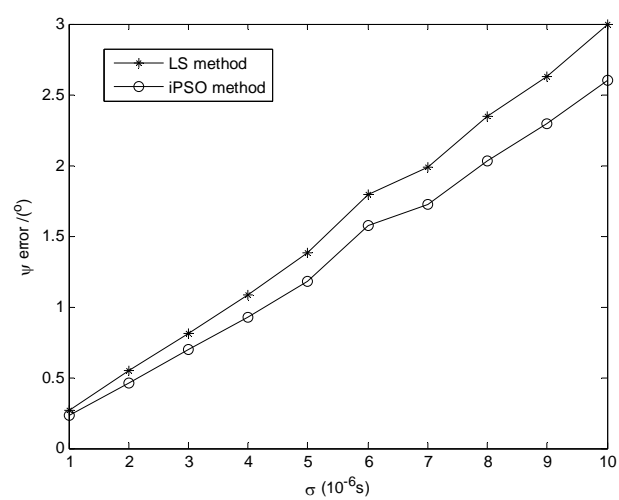

(a) mean of $\varphi$ error

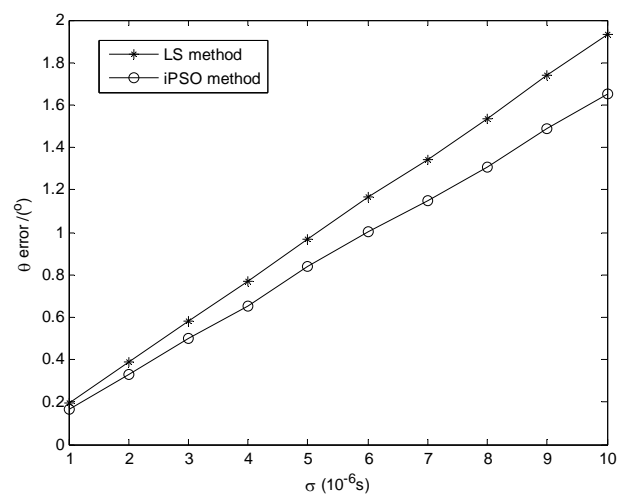

(b) mean of $\theta$ error

FIGURE IV. DOA ESTIMATION ERROR AT DIFFERENT NOISE INTENSITIES

In order to further analyze the performance between the PSO algorithm and the iPSO algorithm, the target DOA is calculated by the PSO algorithm and the iPSO algorithm respectively in the above simulation, and the average fitness values of these two algorithms are analyzed. The result is shown in Figure 5. As can be seen from Figure 5, the iPSO algorithm can achieve convergence faster than the PSO algorithm.

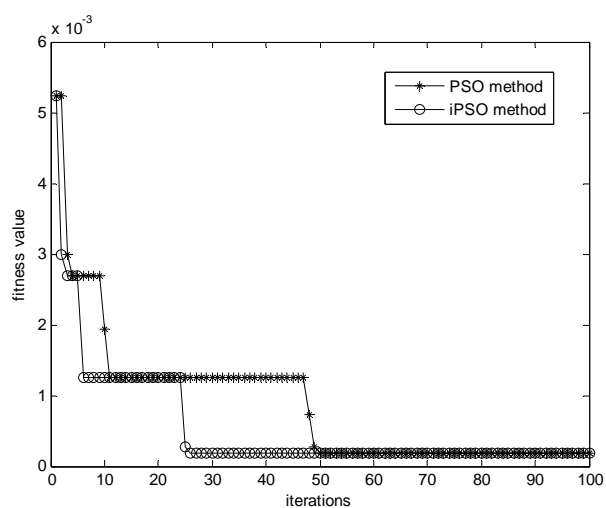

FIGURE V. AVERAGE FITNESS VALUES VARIES WITH NUMBER OF ITERATIONS 


\section{CONCLUSION}

In this paper, an improved PSO algorithm based on regiondivision is proposed. The algorithm divides all particles into two parts and dynamically adjusts the inertia weight and learning factors of the particles to achieve the balance between optimization ability and convergence speed, and introduces adaptive mutation operation to avoid the population falling into local optimum. The proposed algorithm is applied to the DOA estimation of the target by a tetrahedral-based USBL system. Simulation experiments show that the proposed method can effectively improve the accuracy and convergence speed of direction finding.

\section{ACKNOWLEDGMENT}

This research was financially supported by The National Natural Science Foundation of China (grant number: 61371151)

\section{REFERENCES}

[1] Caiti A, Corato F D, Fenucci D, et al. "Experimental results with a mixed USBL/LBL system for AUV navigation". Underwater Communications and Networking. IEEE, 2015: pp.1-4.

[2] Reis, J., Morgado, M., Batista, P., Oliveira, P., \& Silvestre, C. "Design and Experimental Validation of a USBL Underwater Acoustic Positioning System". Sensors, 2016, 16(9).

[3] Biguesh M. "Bearing Estimation Using Time Delays: Optimum Sensor Arrangement and an Efficient Estimator". IEEE Sensors Journal, 2016, 16(18):pp. 6961-6965.

[4] Watson, M., Loggins, C., and Ochi, Y. T. "A new high accuracy supershort baseline (SSBL) system". International Symposium on Underwater Technology. IEEE, 1998: pp. 210-215.

[5] Lima F. V. F. D., and Furukawa C M. "Development and testing of an acoustic positioning system - description and signal processing". Ultrasonics Symposium, 2002. Proceedings. IEEE, 2002. vol.1: pp. 849852

[6] Bingham B., Blair B., and Mindell D. "On the Design of Direct Sequence Spread-Spectrum Signaling for Range Estimation". Oceans. IEEE, 2007: pp. 1-7.

[7] Beaujean P. P. J., Bon A., and An E.. "Motion-compensated acoustic positioning in very shallow waters using spread-spectrum ultra-short baseline array processing". Oceans. IEEE, 2008: pp. 1-7.

[8] Chen H. W., Zhao J. W., and Guo Y. C.. "Acoustic Passive Localization Algorithm Based on a Planar Five-element Array and Its Performance Analysis". Journal of Detection \& Control, 2003, 25(4): pp. 11-16.

[9] Min, Y. U., Hui, J. Y., Feng H. H., and Zhang, X. L.. "Improved Measurement methods for positioning precision of USBL". The Ocean Engineering, 2006, 24(1): pp. 86-91

[10] Xiang, LI, Zhang, D. L., and Sun, D. J.. "Realization of high precision ultra-short baseline positioning system". Computer Engineering and Applications, 2007, 42(24): pp. 176-178.

[11] ARKHIPOV M. "An approach to using basic three-element arrays in tetrahedral-based USBL systems". Oceans - San Diego. IEEE, 2013: pp. 1-8.

[12] ARKHIPOV M. "Designing a USBL system based on a square pyramid array with a complete set of three-element arrays". Oceans. 2012: pp. 1-9.

[13] Zhong F, and Zhou W. "Optimal method for USBL underwater acoustic positioning by combining TDOA and TOA". ACM International Conference on Underwater Networks \& Systems. ACM, 2016:45.

[14] Zaman, F., Qureshi, I. M., Naveed, A., Khan, J. A., and Raja, M. A. Z.. "Amplitude and Directional of Arrival Estimation: Comparison Between Different Techniques". Prog. Electromagn. Res. B, 2012, (39): pp. 319335.
[15] Zaman, F., Qureshi, I. M., Khan, J. A., and Khan, Z. U.. "An Application of Artificial Intelligence for the Joint Estimation of Amplitude and TwoDimensional Direction of Arrival of Far Field Sources Using 2-L-Shape Array". International Journal of Antennas and Propagation,2013,(20135-29), 2013, 2013(7): pp. 718-720.

[16] Lui K. W. K., Zheng J., and So H. C.. "Particle swarm optimization for time-difference-of-arrival based localization". Signal Processing Conference, 2007, European. IEEE, 2008: pp. 414-417.

[17] Cui X., Yu K., and Lu S.. "Evolutionary TDOA-Based Direction Finding Methods With 3-D Acoustic Array". IEEE Transactions on Instrumentation \& Measurement, 2015, 64(9): pp. 2347-2359.

[18] Morgado M., Oliveira P., and Silvestre C.. "Experimental evaluation of a USBL underwater positioning system". In Proceedings of the IEEE ELMAR Conference, Zadar, Croatia, 15-17 September 2010; pp. 485488

[19] Chen, K. S., Yu-Long, R., Dai, L., Lan, Z. G., and Shao, J. S. "Regional-Segmentation Self-Adapting Variation Particle Swarm Optimization". Acta Electronica Sinica, 2017, 45(8): pp. 1849-1855.

[20] Chen M. Y., Zhang C. Y., and Luo C. Y.. "Adaptive evolutionary multiobjective particle swarm optimization algorithm". Control and Decision, 2009, 24(12): pp. 1851-1855.

[21] Feng X. F., and Lv S. F.. "Wireless sensor networks locating algorithm based on RSSI and split-step particle swarm optimization algorithm". Control and Decision, 2014, 29(11): pp. 1966-1972.

[22] Tang K. Z., and Feng J. W., Li F, et al. "Multi-strategy adaptive particle swarm optimization algorithm". Journal of Nanjing Unniversity of Science and Technology, 2017, 41(3): pp. 301-306. 